\title{
Aldosterone Target Neurons in the Nucleus Tractus Solitarius Drive Sodium Appetite
}

\author{
Joel C. Geerling, ${ }^{1}$ William C. Engeland, ${ }^{2}$ Mitsuhiro Kawata, ${ }^{3}$ and Arthur D. Loewy ${ }^{1}$ \\ ${ }^{1}$ Department of Anatomy and Neurobiology, Washington University School of Medicine, St. Louis, Missouri 63110, ${ }^{2}$ Departments of Surgery and \\ Neuroscience, University of Minnesota, Minneapolis, Minnesota 55455, and ${ }^{3}$ Department of Anatomy and Neurobiology, Kyoto Prefectural University of \\ Medicine, Kyoto 602-8566, Japan
}

\begin{abstract}
Sodium appetite can be enhanced by the adrenal steroid aldosterone via an unknown brain mechanism. A novel group of neurons in the nucleus tractus solitarius expresses the enzyme 11- $\beta$-hydroxysteroid dehydrogenase type 2 , which makes them selectively responsive to aldosterone. Their activation parallels sodium appetite in different paradigms of salt loss even in the absence of aldosterone. These unique aldosterone target neurons may represent a previously unrecognized central convergence point at which hormonal and neural signals can be integrated to drive sodium appetite.
\end{abstract}

Key words: aldosterone; ingestive behavior; mineralocorticoid; salt appetite; nucleus of the solitary tract; nucleus tractus solitarius; thirst

\section{Introduction}

Sodium intake is essential for life. When body fluids are lost, blood volume cannot be restored by drinking water alone; sodium must be ingested as well (McCance, 1936; Nose et al., 1988). Sodium deficiency causes severe deficits in growth and reproduction (Fine et al., 1987), and, ultimately, a salt-free diet is lethal (Orent-Keiles et al., 1937).

Sodium deficiency activates a hard-wired brain circuit that produces a voracious appetite for salt. Animals will consume large volumes of highly concentrated saline when they are sodium-deficient (Richter, 1936). Sodium-deficient rats work harder for salt (Wagman, 1963), and their ravenous appetite for salt supersedes robust preferences for sugar and even for directly rewarding brain stimulation (Smith et al., 1968; Conover et al., 1994). Sodium-deficient humans may exhibit sodium appetite as well (Takamata et al., 1994; Kochli et al., 2005).

Aldosterone is secreted from the adrenal cortex in response to sodium deficiency and amplifies sodium intake. Its central site(s) of action may provide clues to the neural circuitry responsible for sodium appetite. Aldosterone is not required for sodium appetite (Richter, 1936), but it enhances salt intake. Although a small dose in adrenalectomized $(\mathrm{adx})$ rats reduces the sodium losses that otherwise would induce sodium appetite (Fregly and Waters, 1966), higher doses of aldosterone and other adrenal mineralocorticoids increase sodium intake even in the absence of sodium deficiency (Rice and Richter, 1943; Wolf, 1965; Wolf and Handal,

Received July 27, 2005; revised Nov. 7, 2005; accepted Nov. 11, 2005.

This work was supported by Grant HL-25449 from the National Heart, Lung, and Blood Institute of National Institutes of Health (A.D.L.), National Science Foundation Grant NSF IBN0112543 (W.C.E.), and American Heart Association Predoctoral Fellowship Award $0510050 Z$ (J.C.G.)

Correspondence should be addressed to Dr. Arthur D. Loewy, Department of Anatomy and Neurobiology, Box 8108, Washington University School of Medicine, 660 South Euclid Avenue, St. Louis, M0 63110. E-mail: loewya@pcg.wustl.edu.

DOI:10.1523/JNEUROSCI.3115-05.2006

Copyright $\odot 2006$ Society for Neuroscience $\quad$ 0270-6474/06/260411-07\$15.00/0
1966). In fact, deoxycorticosterone acetate (DOCA) stimulates a pattern of saline ingestion nearly indistinguishable from adrenalectomy (Stricker et al., 1992). If mineralocorticoids can stimulate sodium intake just like a physiologic deficiency, identifying aldosterone target sites within the brain could provide a key to unraveling the neural circuitry underlying sodium appetite.

Few brain sites contain aldosterone-sensitive cells (Roland et al., 1995; Geerling et al., 2006). Sensitivity to aldosterone requires that cells meet three minimum conditions: (1) they must express the mineralocorticoid receptor (MR); (2) they must express 11- $\beta$-hydroxysteroid dehydrogenase type 2 (HSD2), the enzyme needed to inactivate the abundant adrenal glucocorticoids (corticosterone in rodents, cortisol in humans) that normally saturate the MR, preventing aldosterone from binding (supplemental Fig. 1, available at www.jneurosci. org as supplemental material) (Funder et al., 1988); (3) they must be accessible to aldosterone.

MR expression is found in many sites (Arriza et al., 1988), but only one contains neurons that meet all three criteria. In the nucleus tractus solitarius (NTS) we identified a specialized subpopulation of neurons that express both HSD2 and MR (Geerling et al., 2006). The distribution of these neurons overlaps a blood-brain barrier-deficient (BBB-deficient) zone within the NTS (Gross et al., 1990). This location could allow them special access to blood-borne aldosterone, which has been reported to penetrate the BBB much less than other steroids (Pardridge and Mietus, 1979; Funder and Myles, 1996). These neurons are positioned uniquely to receive convergent neural and hormonal input signals related to sodium deficiency. As the only neurons in the brain readily identifiable by their strong HSD2 immunoreactivity, they are referred to here as the HSD2 neurons.

We tested the HSD2 neurons for functional aldosterone sensitivity and selectivity in vivo. Then we studied their activation in response to diverse stimuli for sodium appetite. 


\section{Materials and Methods}

Experimental protocols were approved by the Washington University School of Medicine Institutional Animal Care and Use Committee and conformed to National Institutes of Health guidelines.

Diet. Rats were provided tap water and PicoLab rodent diet number 20 ( $0.33 \%$ sodium; LabDiet, Richmond, IN) ad libitum, except during specified protocols. For sodium deprivation these were replaced with distilled water $\left(\mathrm{dH}_{2} \mathrm{O}\right)$ and low-sodium chow $(0.01 \% \mathrm{Na}$; number 85292 , HarlanTeklad, Madison, WI). Some sodium repletion control groups received high-sodium chow (8\% Na; number 82050, Harlan-Teklad).

Perfusions and histology. At the end of each experiment the rats (275325 g; male; Sprague Dawley Harlan, Indianapolis, IN) were anesthetized with pentobarbital $(50 \mathrm{mg} / \mathrm{kg})$ and perfused transcardially with isotonic saline, followed by $4 \%$ paraformaldehyde in $0.1 \mathrm{~m}$ sodium phosphate buffer, $\mathrm{pH}$ 7.4. Double-immunofluorescence staining was performed by using a sheep antibody raised against HSD2 (1:40,000; Chemicon, Temecula, CA) (Gomez-Sanchez et al., 2001) combined with a rabbit antibody that specifically labels MR (1:5000) (Ito et al., 2000) or c-Fos (1: 10,000; c-Fos Ab-5 from Oncogene, Cambridge, MA).

MR nuclear translocation experiments. The subcellular (nuclear vs cytoplasmic) localization of the MR within HSD2 neurons was analyzed under four different treatment groups: vehicle, aldosterone, corticosterone, and corticosterone plus aldosterone. First the rats were adrenalectomized bilaterally (under pentobarbital anesthesia) to eliminate endogenous steroid production. Then during this surgery osmotic minipumps were implanted intraperitoneally (Alzet 2ML1, Durect, Cupertino, CA).

Before implantation the minipumps were filled with $150 \mu \mathrm{g} / \mathrm{ml}$ aldosterone (Sigma, St. Louis, MO) or vehicle ( $1 \%$ ethanol in sterile $0.9 \%$ saline). Aldosterone was infused at a rate of $36 \mu \mathrm{g} / \mathrm{d}(1.5 \mu \mathrm{g} / \mathrm{h} ; 10 \mu \mathrm{l} / \mathrm{h}$ for the $2 \mathrm{ML} 1 \mathrm{minipump}$ infusion rate). In pilot tests (using 25-100 $\mu \mathrm{g} / \mathrm{d}$ ) this dose produced blood plasma aldosterone concentrations in the range measured from sodium-depleted rats $(50-100 \mathrm{ng} / \mathrm{dl})$. For the corticosterone-only group of rats a subcutaneous continuous release pellet (Akana et al., 1985), composed of $25 \%$ corticosterone (Sigma) and $75 \%$ cholesterol (Sigma), was inserted during adrenalectomy surgery. This pellet produced plasma concentrations that were 100 -fold greater than the aldosterone infusion, somewhat higher than the average levels in unstressed rats (Akana et al., 1985; Windle et al., 1998).

After $3 \mathrm{~d}$ the rats were reanesthetized, and a 2-4 $\mathrm{ml}$ blood sample was drawn from the left ventricle immediately before transcardial perfusion and centrifuged at $4^{\circ} \mathrm{C}$. Plasma samples were frozen $\left(-80^{\circ} \mathrm{C}\right)$ for subsequent measurement of aldosterone and corticosterone concentrations by radioimmunoassay (RIA), as described by Wotus and Engeland (2003). RIA measurements served as verification that target plasma levels of aldosterone and corticosterone were achieved and that no adrenal tissue remained. Adx vehicle-treated animals were excluded from analysis if residual aldosterone $(>1.25 \mathrm{ng} / \mathrm{dl})$ or corticosterone $(>6.25 \mathrm{ng} / \mathrm{ml})$ production was detected, as were any adx-aldosterone rats in which corticosterone was detected or adx-corticosterone rats with detectable aldosterone.

After double-immunofluorescence staining for HSD2 plus MR, slides were given random code numbers, and a systematic sample of HSD2 neurons from each rat was scored by an observer blinded to treatment conditions. Every HSD2 neuron (green immunofluorescent perikarya) containing a nucleus (a round or ovoid area lacking green immunofluorescence within an HSD2 neuron) in a one-in-five series of $50 \mu \mathrm{m} \mathrm{sec-}$ tions through the NTS $(\approx 150-200$ total neurons in each case) was counted and scored at $400 \times$ magnification for the presence or absence of dense MR immunoreactivity within its nucleus. A distinct nucleolus often was evident within the dense nuclear MR immunoreactivity (red) of cells scored for the presence of nuclear localization.

Sodium appetite experiments. For the first set of dietary sodium deprivation experiments multiple groups of rats $(n=8$ each) were caged individually and provided ad libitum access to low-sodium chow $(0.01 \%$ $\mathrm{Na}$ ) and $\mathrm{dH}_{2} \mathrm{O}$. Four rats from each group were kept on this diet for 2, 3, $4,5,6,7$, or $8 \mathrm{~d}$ (sodium-deprived) while the remaining four were switched to high-sodium chow $(8 \% \mathrm{Na}) 24 \mathrm{~h}$ before perfusion (sodiumreplete controls). Clean cages were provided every day.
Additional groups ( $n=6$ each) initially were provided with $3 \% \mathrm{NaCl}$ solution and $\mathrm{dH}_{2} \mathrm{O}$ in two graduated drinking tubes in addition to lowsodium chow. Their baseline fluid intake volumes were recorded for $3 \mathrm{~d}$, and then the $3 \%$ saline tube was removed. After $8 \mathrm{~d}$ without sodium the $3 \% \mathrm{NaCl}$ tube was returned, and then fluid intake volumes were measured every $30 \mathrm{~min}$ to assess their voluntary sodium ingestion (sodium appetite). Rats were perfused in three groups: $+2,+7$, and $+24 \mathrm{~h}$ of access to $3 \%$ saline. In one additional control experiment overall c-Fos expression in the NTS was measured after $7 \mathrm{~d}$ of sodium deprivation $(n=$ $7)$ versus $7 \mathrm{~d}$ of high-sodium chow $(n=7)$.

For furosemide experiments the rats were injected twice (intraperitoneally) with this diuretic/natriuretic drug (Sigma), spaced $2 \mathrm{~h}$ apart, to ensure maximum volume loss. Urinary sodium loss was not measured directly, but a 10-20 g loss of body weight was confirmed for every rat, indicating $10-20 \mathrm{ml}$ of fluid diuresis. Rats were provided with either low-sodium $(n=5)$ or high-sodium $(n=4)$ chow and distilled water for the next $24 \mathrm{~h}$; a $48 \mathrm{~h}$ test was run in an identical manner.

For DOCA experiments the rats $(n=4)$ were given daily subcutaneous injections of $2 \mathrm{mg}$ of DOCA (Sigma) or vehicle $(0.5 \mathrm{ml}$ vegetable oil; Schnucks Markets, St. Louis, MO). These rats were provided with continuous access to $\mathrm{dH}_{2} \mathrm{O}$ and $3 \%$ saline, and saline intake was monitored daily for $7 \mathrm{~d}$.

For sodium deprivation experiments in adx rats the adx groups $(n=$ 4-6 each) were adrenalectomized bilaterally, whereas control groups ( $n=3-5$ each) were sham-adrenalectomized. During $7 \mathrm{~d}$ of recovery after surgery all rats were provided with a $3 \%$ saline drinking tube in addition to their chow and water bottle.

Saline intake was measured daily during this $7 \mathrm{~d}$ period and used as screening test for complete removal of both adrenal glands (McEwen et al., 1986). Nearly all rats from which two fully encapsulated adrenal glands had been removed completely (verified postoperatively in a dissecting microscope) increased their daily $3 \%$ saline intake to $\sim 15 \mathrm{ml} / \mathrm{d}$ by $7 \mathrm{~d}$ after adrenalectomy, whereas none of the sham-operated control rats drank $>5 \mathrm{ml} / \mathrm{d}$. Adx rats that did not increase their daily intake of $3 \%$ $\mathrm{NaCl}$ to at least $10 \mathrm{ml} / \mathrm{d}$ by postoperative day 7 were excluded from additional analysis; in these few cases a torn or incomplete adrenal gland had been noted immediately after surgery. After this $7 \mathrm{~d}$ recovery/screening period the $3 \% \mathrm{NaCl}$ tubes were removed.

Then sham and adx rats were divided into seven groups ( $n=3-5$ per group) and perfused after dietary sodium deprivation (as above) for 1-6 $\mathrm{d}$ (adx and sham groups) or $7 \mathrm{~d}$ (sham only). At longer sodium deprivation time points $(5-7 \mathrm{~d})$ adx rats became lethargic. None survived through a full $7 \mathrm{~d}$, whereas all sham-adx rats remained healthy until perfusion. An additional adx group $(n=5)$ was sodium-deprived for $3 \mathrm{~d}$ (the time at which HSD2 neuron activation and sodium appetite are both maximal) [Jalowiec and Stricker (1973), their Fig. 6 and the intake data from Table 1] and then provided $24 \mathrm{~h}$ access to $3 \%$ saline to measure the effect of voluntary sodium repletion on HSD2 neuron activity.

Data analysis. For each case the number of HSD2 neurons containing nuclear c-Fos immunoreactivity was divided by the total number of HSD2 neurons to provide a percentage estimate. All data are presented as the group mean \pm SEM. Student's two-tailed $t$ test with a significance level of $p<0.05$ was used for statistical comparisons, except in one experiment (furosemide plus $24 \mathrm{~h}$, for which the Mann-Whitney $U$ test was used; see below).

In the $24 \mathrm{~h}$ furosemide control group (high-sodium chow after diuresis) c-Fos expression was virtually absent within HSD2 neurons (range, $0-2 \%$ ). In contrast, every case in the $24 \mathrm{~h}$ sodium-deprived group (lowsodium chow) was elevated above the control range: three cases exhibited mild $(6.5,7.7,8.9 \%)$ c-Fos expression while two others had much higher levels $(34,37 \%)$, which were within the range of the $48 \mathrm{~h}$ data $(20.0-$ $47.2 \%$ ). Thus $24 \mathrm{~h}$ after diuresis may represent a time point near which activation of the HSD2 neurons increases from a moderately elevated level to one that is sustained for the next day. The high variance in the $24 \mathrm{~h}$ sodium-deprived furosemide group resulted in a nonsignificant difference versus control (high-sodium chow/sodium repletion) by Student's two-tailed $t$ test $(p=0.053)$ despite the obvious qualitative difference and non-overlapping ranges. For this reason, in this one experiment the nonparametric Mann-Whitney $U$ test was used, instead of a two-tailed $t$ 

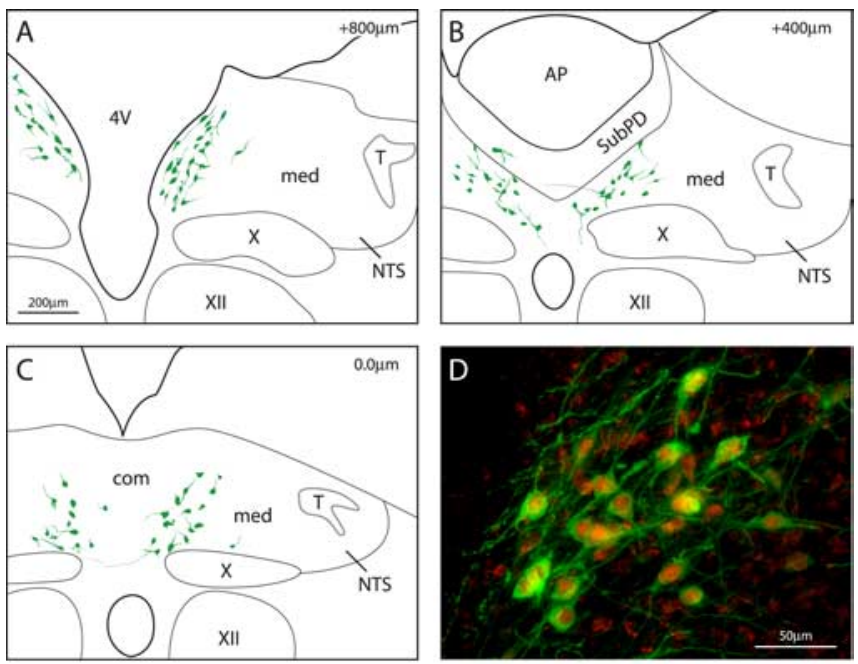

Figure 1. The location of neurons immunoreactive for the enzyme HSD2, which is critical for aldosterone selectivity, is shown at three rostrocaudal levels of the dorsomedial medulla $(\boldsymbol{A}-\boldsymbol{C})$. These neurons (green) lie within the NTS from just rostral to area postrema $(\boldsymbol{A})$ through $\sim 1 \mathrm{~mm}$ caudal (C). Rostrocaudal distances (top right; in micrometers) are relative to the level of calamus scriptorius (C). D, MR immunoreactivity (red) within the nuclei and perinuclear cytoplasm of HSD2-immunoreactive neurons (green) in the NTS from an animal with high plasma aldosterone. AP, Area postrema; 4V, fourth ventricle; med, medial subnucleus of NTS; com, commissural subnucleus of NTS; SubPD, dorsal subpostremal zone; T, solitary tract; $X$, dorsal motor nucleus of the vagus; XII, hypoglossal nucleus.

test, for comparison without assumption about variance and demonstrated a significant difference $(p=0.016)$.

\section{Results}

HSD2 neurons of the NTS are aldosterone-sensitive and aldosterone-selective

The nuclear translocation of the MR was analyzed in vivo to test whether HSD2 neurons in the NTS are aldosterone-sensitive and aldosterone-selective. After the MR binds aldosterone (or corticosterone) in the cytoplasm, the activated ligand-receptor complex enters the nucleus to act as a transcription factor. The utility of MR nuclear translocation as an assay for cellular aldosterone sensitivity and selectivity has been demonstrated previously in vitro (Fejes-Toth et al., 1998; Odermatt et al., 2001). Here we examined MR nuclear translocation in the HSD2 neurons in vivo.

In adx vehicle-infused rats the MR rarely accumulated in the nuclei of HSD2 neurons ( $3.7 \pm 2.1 \% ; n=7$ ) (see Fig. 2, veh). Aldosterone and corticosterone were undetectable in blood plasma samples from these animals. In aldosterone-infused rats, however, a majority of HSD2 neurons exhibited dense nuclear MR immunoreactivity $\left(62.7 \pm 4.7 \% ; p<10^{-7}\right.$ vs vehicle by twotailed $t$ test; $n=13$ ) (Figs. $1 D, 2$, aldo). In these animals the plasma aldosterone concentrations $(74.4 \pm 10.2 \mathrm{ng} / \mathrm{dl})$ approximated the values measured in sodium-deplete rats while corticosterone remained undetectable.

In contrast, corticosterone had only a small effect on MR nuclear translocation in the HSD2 neurons $(19.4 \pm 4.5 \% ; p<0.001$ vs aldo group; $n=4$ ) (Fig. 2, cort) despite plasma levels (7.9 \pm 1.4 $\mu \mathrm{g} / \mathrm{dl})>100$ times higher than the aldosterone concentrations measured in the aldosterone-infused group. The percentage of HSD2 neurons with dense nuclear MR in this group was, nonetheless, elevated above that of vehicle-treated rats $(p=0.015)$. Administration of aldosterone in combination with corticosterone consistently elevated the percentage of HSD2 neurons with dense nuclear MR $\left(75.2 \pm 4.0 \% ; p<10^{-8}\right.$ vs veh; $p<10^{-4}$ vs

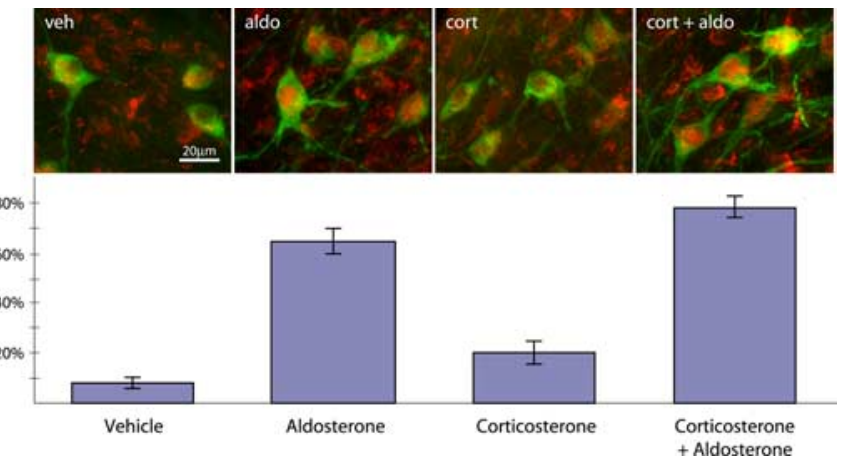

Figure 2. HSD2 neurons are aldosterone-sensitive and aldosterone-selective. The percentage of HSD2-immunoreactive neurons (green) in the NTS containing dense nuclear MR immunoreactivity (red) is shown from adrenalectomized rats that were administered vehicle (veh), aldosterone (aldo), corticosterone (cort), or corticosterone plus aldosterone (cort + aldo). This percentage represents steroid binding and MR translocation from the cytoplasm into the nucleus. Error bars represent SEM.

cort; $n=6)$ (Fig. 2, cort + aldo), but not significantly higher than aldosterone alone $(p=0.12)$.

HSD2 neurons are activated by dietary sodium deprivation Aldosterone can increase sodium intake (Wolf and Handal, 1966), so we hypothesized that the aldosterone-sensitive HSD2 neurons drive sodium appetite. Dietary sodium deprivation was tested first. This noninvasive treatment is a potent stimulus for both aldosterone production and sodium appetite (Contreras and Hatton, 1975). Dietary sodium deprivation results in an appetite that is highly specific for sodium (relative to water), particularly when compared with pharmacological stimuli that often are used in sodium appetite experiments (Prakash and Norgren, 1991).

Rats were deprived of sodium for different lengths of time $(2-8 \mathrm{~d} ; n=4 / \mathrm{d})$, and their HSD2 neurons were analyzed for nuclear c-Fos immunoreactivity. Beginning at $3 \mathrm{~d}$ of sodium deprivation, the HSD2 neurons were activated increasingly (Fig. $3 A, C$ ), as indicated by an increasing percentage with nuclear c-Fos, a marker for neural activity (Hoffman et al., 1993).

Feeding salt to sodium-deprived rats abolished c-Fos expression in the HSD2 neurons. These control rats $(n=4 / \mathrm{d})$ were deprived of sodium for $1-7 \mathrm{~d}$ but then were fed $8 \%$ sodium chow instead of sodium-free chow for $1 \mathrm{~d}$. In every case the HSD2 neurons were virtually devoid of nuclear c-Fos (range, $0-3.8 \%$ ) regardless of the previous duration of sodium deprivation (all $p<0.02$ vs sodium deprivation after $2 \mathrm{~d}$ ) (Fig. 3C).

To test whether the elevation in c-Fos activation during dietary sodium deprivation simply reflected a generalized increase in NTS activity, we compared c-Fos expression between a group of $7 \mathrm{~d}$ sodium-deprived rats $(n=7)$ and a group fed high-sodium chow for $7 \mathrm{~d}(n=7)$. Both the HSD2 neurons and the entire NTS (without respect to neuronal phenotype) were examined in the same set of medullary sections. As before, $7 \mathrm{~d}$ sodium-deprived rats showed higher c-Fos activation in their HSD2 neurons $(46.1 \pm 3.2 \%)$ than sodium-fed rats $\left(0.4 \pm 0.2 \% ; p<10^{-8}\right)$. In the same brainstem sections, however, c-Fos expression throughout the entire NTS was not significantly different $(744.1 \pm 64.2$ sodium-deprived vs $769.1 \pm 65.8$ high-sodium diet; $p=0.83$ ), suggesting that activation within the NTS during dietary sodium deprivation may be a specialized function of the HSD2 neurons. 

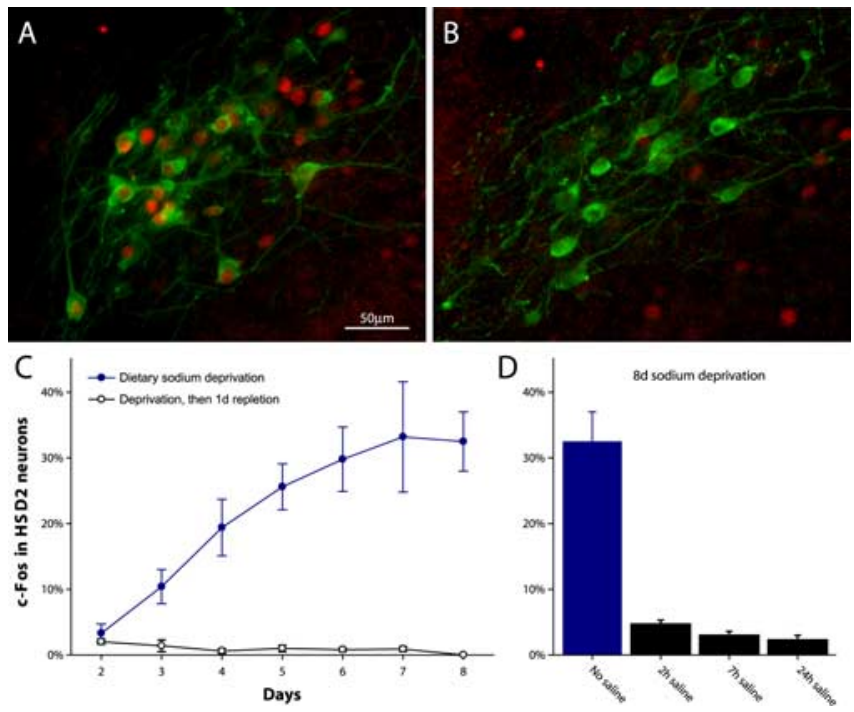

Figure 3. Sodium deprivation increases c-Fos activation in HSD2 neurons in the NTS. A, Many HSD2 neurons (green) contain c-Fos-immunoreactive nuclei (red) after dietary sodium deprivation (7d). $\boldsymbol{B}$, In $6 \mathrm{~d}$ sodium-deprived rats, $1 \mathrm{~d}$ of sodium repletion eliminates c-Fos expression in HSD2 neurons. C, The percentage of c-Fos-positive HSD2 neurons increases with time in sodium-deprived intact rats (filled blue circles), but $1 \mathrm{~d}$ of high-sodium diet consistently abolishes this activation (open black circles). $\boldsymbol{D}$, The elevation in c-Fos-positive HSD2 neurons $(32.5 \pm 4.5 \%)$ caused by $8 \mathrm{~d}$ of dietary sodium deprivation is nearly abolished within $2 \mathrm{~h}$ of access to $3 \%$ saline. Error bars represent SEM.

\section{Rapid inactivation of HSD2 neurons by voluntary sodium ingestion}

Additional groups of rats were provided voluntary access to concentrated saline $(3 \%=0.5 \mathrm{M} \mathrm{NaCl})$, instead of high-sodium chow, after $8 \mathrm{~d}$ of dietary sodium deprivation. These tests were conducted, first, to verify that our sodium deprivation protocol increased sodium appetite, and, second, to determine whether the inactivation of HSD2 neurons by salt intake occurs immediately or after delayed postingestive effects.

Rats perfused after $8 \mathrm{~d}$ dietary sodium deprivation, without access to saline, showed a high percentage of activated HSD2 neurons ( $32.5 \pm 4.5 \%$ c-Fos expression; $n=6$ ). As shown in Figure $3 D$, however, the HSD2 neurons were inactivated significantly after just $2 \mathrm{~h}$ of access to $3 \% \mathrm{NaCl}(4.7 \pm 0.6 \%$ nuclear c-Fos; $p=0.0001$ vs $8 \mathrm{~d}$ of sodium deprivation without saline access; $n=6)$. During this $2 \mathrm{~h}$ access period these rats drank $7.5 \pm$ $0.6 \mathrm{ml}$ of $3 \%$ saline ( $p<10^{-7}$ vs predeprivation average, $1.67 \pm$ $0.4 \mathrm{ml} / 24 \mathrm{~h})$, primarily within the first $30 \mathrm{~min}(6.7 \pm 0.7 \mathrm{ml})$.

Similar inactivation of the HSD2 neurons was seen after $7 \mathrm{~h}$ of access to saline ( $3.0 \pm 0.6 \% \mathrm{c}$-Fos expression; $n=6 ; p<10^{-4}$ vs no saline access and $p=0.09$ vs $2 \mathrm{~h}$ group, not significant). Total $3 \%$ saline intake $(6.8 \pm 1.3 \mathrm{ml})$ and initial $30 \mathrm{~min}$ intake $(5.3 \pm$ $0.8 \mathrm{ml}$ ) in the $7 \mathrm{~h}$ group were not significantly different from the $2 \mathrm{~h}$ group ( $p=0.64$ and $p=0.23$, respectively).

Rats given $24 \mathrm{~h}$ access to saline $(n=5)$ drank a similar amount during the first $30 \mathrm{~min}$ of access $(6.9 \pm 1.3 \mathrm{ml} ; p>0.3$ vs both 2 and $7 \mathrm{~h}$ ) and drank only an additional $4.9 \pm 0.8 \mathrm{ml}$ over the subsequent $23.5 \mathrm{~h}$. The HSD2 neurons remained inactivated at this time point $(2.3 \pm 0.7 \% \mathrm{c}$-Fos expression; $p=0.0002$ vs $8 \mathrm{~d}$ of deprivation), just like rats switched to $8 \%$ sodium chow after sodium deprivation (Fig. $3 \mathrm{~B}, \mathrm{C}$ ). In this $24 \mathrm{~h} 3 \%$ saline access group c-Fos expression in the HSD2 neurons was slightly lower than in the 2 h group ( $p=0.04)$, but not significantly lower than the 7 hroup $(p=0.48)$.

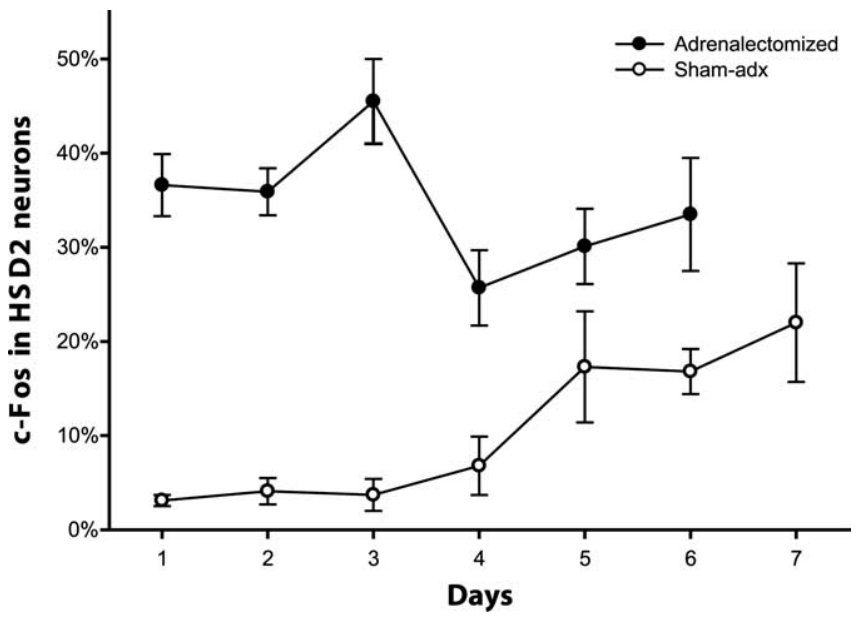

Figure 4. When adrenalectomized animals (filled circles) are sodium-deprived, HSD2 neurons show elevated c-Fos activation by just $1 \mathrm{~d}$ after sodium is removed. This elevated activation peaks at day 3 and then parallels the pattern of adrenal-intact (sham-operated) rats. In shamadrenalectomized (sham-adx) control rats subjected to the same dietary sodium deprivation protocol (open circles), HSD2 neuron activation was delayed, similar to intact rats. Error bars represent SEM.

\section{Activation of HSD2 neurons by diuresis and DOCA}

Diuresis produced by the drug furosemide, combined with shortterm dietary sodium deprivation, is a method frequently used to evoke sodium appetite (Jalowiec, 1974). The percentage of c-Fosexpressing HSD2 neurons was elevated consistently when furosemide diuresis was followed by a 24 or $48 \mathrm{~h}$ sodium-free diet (18.7 \pm 6.8 and $29.4 \pm 4.7 \% ; n=5$ each). Feeding furosemidetreated rats high-sodium instead of low-sodium chow prevented this c-Fos activation at both $24 \mathrm{~h}(0.8 \pm 0.2 \% ; p<0.02$ vs $24 \mathrm{~h}$ of sodium-free diet; $n=4)$ and $48 \mathrm{~h}(1.5 \pm 0.5 \% ; p<0.002$ vs $48 \mathrm{~h}$ of sodium-free diet; $n=4$ ).

We also tested whether HSD2 neurons are stimulated by the aldosterone analog DOCA, which dramatically increases sodium appetite even in the absence of a sodium deficit (Rice and Richter, 1943; Wolf, 1965). Vehicle-treated control animals drank very little $3 \%$ saline $(1.4 \pm 0.2 \mathrm{ml} / \mathrm{d}$ by $7 \mathrm{~d} ; n=4)$, and their HSD2 neurons were not activated ( $2.2 \pm 0.5 \%$ c-Fos expression; $n=4)$. DOCA ( $2 \mathrm{mg} / \mathrm{d}$ ) induced an enormous daily intake of $3 \%$ saline $\left(23.8 \pm 0.5 \mathrm{ml} / \mathrm{d}\right.$ by $7 \mathrm{~d} ; p<10^{-7}$ vs veh; $\left.n=4\right)$, and $c$-Fos expression was elevated in the HSD2 neurons from these animals (20.9 $\pm 2.5 \% ; p<0.001$ vs veh) despite their sodium consumption.

\section{Activation by sodium deficiency without aldosterone}

We hypothesized that aldosterone is necessary for HSD2 neuron activation during sodium deficiency. Rats were adrenalectomized to eliminate aldosterone production and then were sodiumdeprived for $1-6 \mathrm{~d}(n=3-5 / \mathrm{d})$. Contrary to our hypothesis, c-Fos expression in the HSD2 neurons was increased greatly after just $1 \mathrm{~d}$ of sodium deprivation $(36.6 \pm 3.3 \% ; n=6)$ and remained elevated through $6 \mathrm{~d}$ (Fig. 4), after which time continued deprivation was uniformly lethal. In contrast, sham-operated control rats $(n=3-4 / \mathrm{d})$ exhibited a delayed time course of c-Fos activation in these neurons, similar to intact rats. The percentage of HSD2 neurons with nuclear c-Fos was higher in adx rats than in sham-operated rats at every time point. This difference was statistically significant for days $1-4(p<0.02$ each), but not on days $5-6(p=0.16 ; p=0.051)$.

Despite this rapid and sustained activation the HSD2 neurons 
were still inactivated by saline ingestion. An additional group of adx rats that were sodium-deprived for $3 \mathrm{~d}(n=5)$ voluntarily drank a large volume of $3 \%$ saline $(28.5 \pm 0.8 \mathrm{ml}$ over $24 \mathrm{~h}$ ), confirming their robust sodium appetite (Jalowiec and Stricker, 1973). After $24 \mathrm{~h}$ of saline access the HSD2 neurons were inactivated in these rats $(2.9 \pm 1.4 \% ; p=0.01$ vs $3 \mathrm{~d}$ of deprivation without saline access; $n=5)$.

\section{Discussion}

The HSD2 neurons of the NTS represent the only identified CNS neurons that are both sensitive to aldosterone and activated during sodium appetite. Aldosterone-sensitive cells may exist in the subcommissural organ and the ventromedial hypothalamic nucleus (Roland et al., 1995; Geerling et al., 2006), but we and others did not detect elevated MR expression in either of these sites (Arriza et al., 1988; Geerling et al., 2006). Also, we did not find evidence for elevated c-Fos expression in these two sites in the experiments described above (data not shown). The experimental results described here suggest that the HSD2 neurons may even play an important role in driving sodium appetite in the absence of aldosterone.

\section{HSD2 neurons of the NTS are aldosterone-sensitive and aldosterone-selective}

Once we identified this putative aldosterone-sensitive group of neurons (Geerling et al., 2006), it became important to determine whether they are accessible to circulating aldosterone in vivo. Relative to other steroids, blood-borne aldosterone has a reduced ability to enter the brain (Pardridge and Mietus, 1979; Funder and Myles, 1996). However, the HSD2 neurons are located in a restricted NTS subregion with a diminished BBB (Gross et al., 1990).

In the HSD2 neurons the nuclear translocation of the MR was increased dramatically by systemic infusion of aldosterone (Fig. 2, aldo). Very little MR nuclear translocation occurs spontaneously (Fejes-Toth et al., 1998), so these data show that physiologic levels of circulating aldosterone can influence the HSD2 neurons.

Their expression of HSD2 predicted that these neurons inactivate glucocorticoids. This inactivation was evident from the significantly reduced ability of corticosterone, at a concentration 100 -fold higher than aldosterone, to increase the nuclear translocation of the MR (Fig. 2, cort). Likewise, the addition of a 100 -fold higher level of corticosterone did not alter MR nuclear translocation significantly in response to aldosterone infusion (Fig. 2, cort + aldo). Corticosterone and aldosterone have equivalent MR binding affinities (Sheppard and Funder, 1987), and in cells without HSD2 a $1 \mathrm{~nm}$ concentration of either steroid induces equivalent MR nuclear translocation (Fejes-Toth et al., 1998).

The small but significant increase in MR nuclear translocation that occurred in response to corticosterone treatment was unexpected (Fig. 2, cort). However, it has been shown that a minor amount of this steroid escapes inactivation by HSD2 (Funder and Myles, 1996), which could be attributable to the lower affinity of corticosterone for this enzyme $(\sim 25 \mathrm{~nm})$ (Naray-Fejes-Toth et al., 1998) relative to the MR ( $<1 \mathrm{~nm})$ (Sheppard and Funder, 1987). Corticosteroid-binding globulin (CBG) reduces the effective circulating concentration of corticosterone, but this effect would not, on its own, explain its reduced potency relative to aldosterone. Even after 95\% sequestration by CBG the free concentration of corticosterone would have remained fivefold higher than aldosterone in these experiments.

\section{HSD2 neurons signal sodium depletion}

Initially, we had predicted that the HSD2 neurons drive the increase in blood pressure that occurs during central infusion of aldosterone (Gomez-Sanchez, 1986). However, the lack of a significant interaction between aldosterone and corticosterone as measured by MR nuclear translocation in the HSD2 neurons (Fig. 2) stood in sharp contrast to the ability of corticosterone to inhibit the effect of aldosterone on blood pressure (GomezSanchez et al., 1990). Blockade by corticosterone suggests that the pressor effect of aldosterone involves cells that express the MR, but not HSD2. Also, viral and conventional neural-tracing experiments have failed to show a linkage between the HSD2 neurons and the autonomic nervous system (our unpublished observations). For these reasons we considered the alternative possibility that HSD2 neurons drive sodium appetite, not blood pressure.

In every salt appetite paradigm we tested, the HSD2 neurons were activated: dietary sodium deprivation, furosemide diuresis, high-dose DOCA, and adrenalectomy. Then, similar to sodium appetite, they were inactivated rapidly and consistently when sodium was consumed (Fig. 3D). Also like sodium appetite, their activation by sodium deficiency did not require aldosterone (Fig. 4).

Sodium appetite and c-Fos activation in the HSD2 neurons show remarkably similar time courses. During dietary sodium deprivation most sodium losses occur within the first $1-2 \mathrm{~d}$, followed by near-total sodium retention mediated by aldosterone (Jalowiec and Stricker, 1973; Contreras and Hatton, 1975). However, a detectable increase in sodium appetite occurs only after 3-4 d (Jalowiec and Stricker, 1973), after which salt intake becomes maximal by 7-10 d (Contreras and Hatton, 1975; Stricker et al., 1991).

HSD2 neuron activation follows an identical time course (Fig. 3 ). Even more striking is the similarity between the activation of HSD2 neurons and the increase in salt ingestion observed after only 1-4 d of dietary sodium deprivation in adx rats [Jalowiec and Stricker (1973), compare their Table 1 with our Fig. 4].

Dietary sodium deprivation noninvasively produces a true physiologic sodium deficit (Contreras and Hatton, 1975; Stricker et al., 1991) and an appetite that is highly specific for sodium (Prakash and Norgren, 1991), but it has not been used in previous Fos studies involving sodium balance. Also, unlike previous Fos studies (Rowland et al., 1996; Houpt et al., 1998; Thunhorst et al., 1998), the present experiments were focused on a specific neuronal phenotype. A profound change in c-Fos expression was observed in the subgroup of NTS neurons that express HSD2 (Fig. 3 ), but when c-Fos-positive nuclei were counted throughout the entire NTS, there was no consistent association with sodium status (see Results). This problem may have limited previous studies that considered Fos location, but not the specific neuronal phenotypes involved (Houpt et al., 1998; Thunhorst et al., 1998).

Many previous studies of sodium appetite have focused on sites in the anteroventral third ventricle (AV3V) region despite the persistence of sodium appetite after large electrolytic lesions here (Bealer and Johnson, 1979) and even though neurons in this region are activated long before sodium appetite, with a time course similar to thirst (Rowland and Morian, 1999). HSD2 expression is not found in this region (Roland et al., 1995; Geerling et al., 2006), suggesting that these sites are not aldosterone-sensitive.

Previously, it was proposed that stimulation of sodium intake by mineralocorticoids is an indirect effect because of an increase in angiotensin II (AII) receptor expression in AV3V sites (King et al., 1988) and/or reduced release of the peptide transmitter oxytocin by neurons in the hypothalamus (Roesch et al., 2001). However, HSD2 is not expressed in hypothalamic sites that are 
activated by AII or that produce oxytocin (Roland et al., 1995; Geerling et al., 2006). The identification of aldosterone-sensitive neurons in the NTS, with activity directly correlated with sodium appetite, offers a more direct route for the enhancement of sodium appetite by mineralocorticoids, although these other modes of action may be involved as well.

\section{HSD2 neurons as a neuroanatomical link to sodium appetite}

A number of previous findings support the idea that there is a relationship between the HSD2 neurons and sodium appetite. For example, HSD2 inhibition by glycyrrhetinic acid (the blood pressureincreasing constituent of licorice), allowing unrestricted MR activation by glucocorticoids, produces an adrenal steroiddependent increase in salt ingestion despite simultaneous overretention of sodium by the kidney (Cooney and Fitzsimons, 1996).

Also, the HSD2 neurons lie immediately ventral to the area postrema, which innervates the NTS and exerts a powerful inhibitory influence on sodium appetite (Contreras and Stetson, 1981). Destruction of the area postrema (without extensive damage to the underlying NTS) causes an enormous daily sodium intake without necessarily altering the increase in salt intake that occurs in response to sodium deficiency (Edwards et al., 1993). This effect depends on the integrity of the underlying NTS, where the HSD2 neurons are located (Curtis et al., 1999). Coupled with the present data, these findings raise the possibility that sodium appetite is driven by the HSD2 neurons but suppressed tonically by neurons in the overlying area postrema.

Our neural tracing studies have revealed that the HSD2 neurons primarily innervate rostral brain sites that previously have been implicated in sodium appetite (our unpublished observations), including subnuclei within the pontine parabrachial region (Scalera et al., 1995) and the bed nucleus of the stria terminalis (Reilly et al., 1994; Zardetto-Smith et al., 1994). It also should be noted that small NTS lesions, lateral to the HSD2 neurons and area postrema, eliminate baroreflex regulation of blood pressure and heart rate yet leave sodium appetite intact (Schreihofer et al., 1999). To our knowledge sodium appetite has not been tested in animals with lesions restricted to the more medial NTS region containing the HSD2 neurons.

\section{Neural and hormonal integration by the HSD2 neurons}

The HSD2 neurons are positioned optimally to integrate peripheral hormonal and neural signals related to sodium deficiency. They are the primary mineralocorticoid target neurons in the brain (Geerling et al., 2006), and they are activated in parallel with sodium appetite during high-dose mineralocorticoid (DOCA) treatment as well as dietary sodium deprivation, which increases aldosterone production. More importantly, they are still activated by sodium deprivation after adrenalectomy, which eliminates aldosterone production (Fig. 4). This finding indicates that the HSD2 neurons receive information about sodium deficiency from other sources in addition to aldosterone. It is not yet clear whether they are sensitive to other hormonal stimuli such as AII, but the HSD2 neurons may be activated during sodium deficiency by synaptic inputs.

Because of their location in the NTS, the viscerosensory nucleus of the brain, the HSD2 neurons may receive neural inputs related to peripheral cardiovascular changes. The vagus nerve provides synaptic input to this part of the NTS (Hamilton and Norgren, 1984), so these neurons conceivably could receive direct or indirect inputs that play a role in sodium homeostasis. Consequently, the HSD2 neurons may represent a unique central site for the convergence of neural and humoral signals of sodium deficiency.

\section{Summary}

Convergent functional and neuroanatomical evidence suggests that the HSD2 neurons play a central role in driving sodium appetite. They produce an output signal that represents the need for sodium, likely influencing rostral appetitive sites in the brain. We propose that the HSD2-expressing aldosterone target neurons of the NTS drive sodium appetite.

\section{References}

Akana SF, Cascio CS, Shinsako J, Dallman MF (1985) Corticosterone: narrow range required for normal body and thymus weight and ACTH. Am J Physiol 249:R527-R532.

Arriza JL, Simerly RB, Swanson LW, Evans RM (1988) The neuronal mineralocorticoid receptor as a mediator of glucocorticoid response. Neuron 1:887-900.

Bealer SL, Johnson AK (1979) Sodium consumption following lesions surrounding the anteroventral third ventricle. Brain Res Bull 4:287-290.

Conover KL, Woodside B, Shizgal P (1994) Effects of sodium depletion on competition and summation between rewarding effects of salt and lateral hypothalamic stimulation in the rat. Behav Neurosci 108:549-558.

Contreras RJ, Hatton GI (1975) Gustatory adaptation as an explanation for dietary-induced sodium appetite. Physiol Behav 15:569-576.

Contreras RJ, Stetson PW (1981) Changes in salt intake lesions of the area postrema and the nucleus of the solitary tract in rats. Brain Res 211:355-366.

Cooney AS, Fitzsimons JT (1996) Increased sodium appetite and thirst in rat induced by the ingredients of liquorice, glycyrrhizic acid and glycyrrhetinic acid. Regul Pept 66:127-133.

Curtis KS, Huang W, Sved AF, Verbalis JG, Stricker EM (1999) Impaired osmoregulatory responses in rats with area postrema lesions. Am J Physiol 277:R209-R219.

Edwards GL, Beltz TG, Power JD, Johnson AK (1993) Rapid-onset "needfree" sodium appetite after lesions of the dorsomedial medulla. Am J Physiol 264:R1242-R1247.

Fejes-Toth G, Pearce D, Naray-Fejes-Toth A (1998) Subcellular localization of mineralocorticoid receptors in living cells: effects of receptor agonists and antagonists. Proc Natl Acad Sci USA 95:2973-2978.

Fine BP, Ty A, Lestrange N, Levine OR (1987) Sodium deprivation growth failure in the rat: alterations in tissue composition and fluid spaces. J Nutr 117:1623-1628.

Fregly MJ, Waters IW (1966) Effect of mineralocorticoids on spontaneous sodium chloride appetite of adrenalectomized rats. Physiol Behav 1:65-74.

Funder J, Myles K (1996) Exclusion of corticosterone from epithelial mineralocorticoid receptors is insufficient for selectivity of aldosterone action: in vivo binding studies. Endocrinology 137:5264-5268.

Funder JW, Pearce PT, Smith R, Smith AI (1988) Mineralocorticoid action: target tissue specificity is enzyme, not receptor, mediated. Science 242:583-585.

Geerling JC, Kawata M, Loewy AD (2006) Aldosterone-sensitive neurons in the rat CNS. J Comp Neurol 494:515-527.

Gomez-Sanchez EP (1986) Intracerebroventricular infusion of aldosterone induces hypertension in rats. Endocrinology 118:819-823.

Gomez-Sanchez EP, Venkataraman MT, Thwaites D, Fort C (1990) ICV infusion of corticosterone antagonizes ICV-aldosterone hypertension. Am J Physiol 258:E649-E653.

Gomez-Sanchez EP, Ganjam V, Chen YJ, Liu Y, Clark SA, Gomez-Sanchez CE (2001) The $11 \beta$-hydroxysteroid dehydrogenase 2 exists as an inactive dimer. Steroids 66:845-848.

Gross PM, Wall KM, Pang JJ, Shaver SW, Wainman DS (1990) Microvascular specializations promoting rapid interstitial solute dispersion in nucleus tractus solitarius. Am J Physiol 259:R1131-R1138.

Hamilton RB, Norgren R (1984) Central projections of gustatory nerves in the rat. J Comp Neurol 222:560-577.

Hoffman GE, Smith MS, Verbalis JG (1993) c-Fos and related immediate early gene products as markers of activity in neuroendocrine systems. Front Neuroendocrinol 14:173-213.

Houpt TA, Smith GP, Joh TH, Frankmann SP (1998) c-Fos-like immunoreactivity in the subfornical organ and nucleus of the solitary tract following salt intake by sodium-depleted rats. Physiol Behav 63:505-510.

Ito T, Morita N, Nishi M, Kawata M (2000) In vitro and in vivo immunocy- 
tochemistry for the distribution of mineralocorticoid receptor with the use of specific antibody. Neurosci Res 37:173-182.

Jalowiec JE (1974) Sodium appetite elicited by furosemide: effects of differential dietary maintenance. Behav Biol 10:313-327.

Jalowiec JE, Stricker EM (1973) Sodium appetite in adrenalectomized rats following dietary sodium deprivation. J Comp Physiol Psychol 82:66-77.

King SJ, Harding JW, Moe KE (1988) Elevated salt appetite and brain binding of angiotensin II in mineralocorticoid-treated rats. Brain Res 448:140-149.

Kochli A, Tenenbaum-Rakover Y, Leshem M (2005) Increased salt appetite in patients with congenital adrenal hyperplasia 21-hydroxylase deficiency. Am J Physiol Regul Integr Comp Physiol 288:R1673-R1681.

McCance RA (1936) Experimental human salt deficiency. Lancet $1: 823-830$

McEwen BS, Lambdin LT, Rainbow TC, De Nicola AF (1986) Aldosterone effects on salt appetite in adrenalectomized rats. Neuroendocrinology 43:38-43.

Naray-Fejes-Toth A, Colombowala IK, Fejes-Toth G (1998) The role of $11 \beta$-hydroxysteroid dehydrogenase in steroid hormone specificity. J Steroid Biochem Mol Biol 65:311-316.

Nose H, Mack GW, Shi XR, Nadel ER (1988) Role of osmolality and plasma volume during rehydration in humans. J Appl Physiol 65:325-331.

Odermatt A, Arnold P, Frey FJ (2001) The intracellular localization of the mineralocorticoid receptor is regulated by $11 \beta$-hydroxysteroid dehydrogenase type 2. J Biol Chem 276:28484-28492.

Orent-Keiles E, Robinson A, McCollum EV (1937) The effects of sodium deprivation on the animal organism. Am J Physiol 119:651-661.

Pardridge WM, Mietus LJ (1979) Transport of steroid hormones through the rat blood-brain barrier. Primary role of albumin-bound hormone. J Clin Invest 64:145-154.

Prakash MR, Norgren R (1991) Comparing salt appetites: induction with intracranial hormones or dietary sodium restriction. Brain Res Bull 27:397-401.

Reilly JJ, Maki R, Nardozzi J, Schulkin J (1994) The effects of lesions of the bed nucleus of the stria terminalis on sodium appetite. Acta Neurobiol Exp (Wars) 54:253-257.

Rice KK, Richter CP (1943) Increased sodium chloride and water intake of normal rats treated with desoxycorticosterone acetate. Endocrinology 33:106-115.

Richter CP (1936) Increased salt appetite in adrenalectomized rats. Am J Physiol 115:155-161.

Roesch DM, Blackburn-Munro RE, Verbalis JG (2001) Mineralocorticoid treatment attenuates activation of oxytocinergic and vasopressinergic neurons by ICV ANG II. Am J Physiol Regul Integr Comp Physiol 280:R1853-R1864.

Roland BL, Li KX, Funder JW (1995) Hybridization histochemical localiza- tion of $11 \beta$-hydroxysteroid dehydrogenase type 2 in rat brain. Endocrinology 136:4697-4700.

Rowland NE, Morian KR (1999) Roles of aldosterone and angiotensin in maturation of sodium appetite in furosemide-treated rats. Am J Physiol 276:R1453-R1460.

Rowland NE, Fregly MJ, Han L, Smith G (1996) Expression of Fos in rat brain in relation to sodium appetite: furosemide and cerebroventricular renin. Brain Res 728:90-96.

Scalera G, Spector AC, Norgren R (1995) Excitotoxic lesions of the parabrachial nuclei prevent conditioned taste aversions and sodium appetite in rats. Behav Neurosci 109:997-1008.

Schreihofer AM, Anderson BK, Schiltz JC, Xu L, Sved AF, Stricker EM (1999) Thirst and salt appetite elicited by hypovolemia in rats with chronic lesions of the nucleus of the solitary tract. Am J Physiol 276:R251-R258.

Sheppard KE, Funder JW (1987) Equivalent affinity of aldosterone and corticosterone for type I receptors in kidney and hippocampus: direct binding studies. J Steroid Biochem 28:737-742.

Smith Jr MH, Holman GL, Fortune KH (1968) Sodium need and sodium consumption. J Comp Physiol Psychol 65:33-37.

Stricker EM, Thiels E, Verbalis JG (1991) Sodium appetite in rats after prolonged dietary sodium deprivation: a sexually dimorphic phenomenon. Am J Physiol 260:R1082-R1088.

Stricker EM, Gannon KS, Smith JC (1992) Salt appetite induced by DOCA treatment or adrenalectomy in rats: analysis of ingestive behavior. Physiol Behav 52:793-802.

Takamata A, Mack GW, Gillen CM, Nadel ER (1994) Sodium appetite, thirst, and body fluid regulation in humans during rehydration without sodium replacement. Am J Physiol 266:R1493-R1502.

Thunhorst RL, Xu Z, Cicha MZ, Zardetto-Smith AM, Johnson AK (1998) Fos expression in rat brain during depletion-induced thirst and salt appetite. Am J Physiol 274:R1807-R1814.

Wagman W (1963) Sodium chloride deprivation: development of sodium chloride as a reinforcement. Science 140:1403-1404.

Windle RJ, Wood SA, Shanks N, Lightman SL, Ingram CD (1998) Ultradian rhythm of basal corticosterone release in the female rat: dynamic interaction with the response to acute stress. Endocrinology 139:443-450.

Wolf G (1965) Effect of deoxycorticosterone on sodium appetite of intact and adrenalectomized rats. Am J Physiol 208:1281-1285.

Wolf G, Handal PJ (1966) Aldosterone-induced sodium appetite: doseresponse and specificity. Endocrinology 78:1120-1124.

Wotus C, Engeland WC (2003) Differential regulation of adrenal corticosteroids after restriction-induced drinking in rats. Am J Physiol Regul Integr Comp Physiol 284:R183-R191.

Zardetto-Smith AM, Beltz TG, Johnson AK (1994) Role of the central nucleus of the amygdala and bed nucleus of the stria terminalis in experimentally induced salt appetite. Brain Res 645:123-134. 